\title{
Scleraxis is Required for Differentiation of the Stapedius and Tensor Tympani Tendons of the Middle Ear
}

\author{
Lingyan Wang ${ }^{1}$, Chris S. Bresee ${ }^{1}$, Han Jiang ${ }^{1}$, Wenxuan He ${ }^{1}$, Tianying Ren ${ }^{1}$, Ronen Schweitzer ${ }^{2}$, \\ AND JOHN V. BRIGANDE ${ }^{1}$ \\ ${ }^{1}$ Oregon Hearing Research Center, Oregon Health E Science University, 3181 SW Sam Jackson Park Road/NRC04, Portland, \\ OR 97239, USA \\ ${ }^{2}$ Research Division, Shriners Hospital for Children, Portland, OR 97239, USA
}

Received: 14 January 2011; Accepted: 22 February 2011; Online publication: 12 March 2011

\begin{abstract}
Scleraxis $(S c x)$ is a basic helix-loop-helix transcription factor expressed in tendon and ligament progenitor cells and the differentiated cells within these connective tissues in the axial and appendicular skeleton. Unexpectedly, we found expression of the $S c x$ transgenic reporter mouse, Scx-GFP, in interdental cells, sensory hair cells, and cochlear supporting cells at embryonic day 18.5 (E18.5). We evaluated $S c x-n u l l$ mice to gain insight into the function of $S c x$ in the inner ear. Paradoxical hearing loss was detected in Scx-nulls, with $\sim 50 \%$ of the mutants presenting elevated auditory thresholds. However, Scx-null mice have no obvious, gross alterations in cochlear morphology or cellular patterning. Moreover, we show that the elevated auditory thresholds correlate with middle ear infection. Laser interferometric measurement of sound-induced malleal movements in the infected Scx-nulls demonstrates increased impedance of the middle ear that accounts for the hearing loss observed. The vertebrate middle ear transmits vibrations of the tympanic membrane to the cochlea. The tensor tympani and stapedius muscles insert into the malleus and stapes via distinct tendons and mediate the middle ear muscle reflex that in part protects the inner ear from noise-induced damage. Nothing, however, is known about the development and function of these tendons. Scx is expressed in tendon progenitors at E14.5 and differentiated teno-
\end{abstract}

Lingyan Wang and Chris S. Bresee contributed equally to this work.

Correspondence to: John V. Brigande · Oregon Hearing Research Center · Oregon Health \& Science University · 3181 SW Sam Jackson Park Road/NRC04, Portland, OR 97239, USA. Telephone: +1-503-4942933; fax: +1-503-4945656; email: brigande@ohsu.edu cytes of the stapedius and tensor tympani tendons at E16.5-18.5. Scx-nulls have dramatically shorter stapedius and tensor tympani tendons with altered extracellular matrix consistent with abnormal differentiation in which condensed tendon progenitors are inefficiently incorporated into the elongating tendons. Scx-GFP is the first transgenic reporter that identifies middle ear tendon lineages from the time of their formation through complete tendon maturation. Scx-null is the first genetically defined mouse model for abnormal middle ear tendon differentiation. $S c x$ mouse models will facilitate studies of tendon and muscle formation and function in the middle ear.

Keywords: middle ear muscle reflex, tendon, tenocytes, laser interferometry, otitis media

\section{INTRODUCTION}

The special sense of hearing requires functional integration of the outer, middle, and inner ears (Hudspeth 1997). Sound energy is collected and focused by the auricle and external acoustic meatus to efficiently vibrate the tympanic membrane. Movement of the tympanic membrane transmits force sequentially to the three bones of the ossicular chain: the malleus, incus, and stapes. The footplate of the stapes impinges upon the oval window of the cochlea and transmits energy to the cochlear partition that will ultimately displace sensory hair cell bundles, the first step in mechanotransduction (Gillespie and Muller 2009). The movement of the middle ear ossicles is modulated by the tensor tympani and stapedius muscles which insert via tendons into bony 
prominences on the malleus and stapes, respectively. The middle ear muscle reflex involves contraction of the tensor tympani and stapedius muscles to modulate mechanical impedance of the middle ear and, in part, shield the inner ear from intense, damaging sound (Mukerji et al. 2010). Progress in understanding middle ear tendon formation and function has been hampered by the absence of a suitable reporter to facilitate dynamic analyses in vivo. Indeed, middle ear tendons and their associated musculature have been largely ignored in developmental studies of the middle ear (Mallo 2001, 2003).

Scx is a basic helix-loop-helix (bHLH) transcription factor with distinct expression in a number of tissues including the musculoskeletal system, epididymi, testes, adrenal gland, kidney, lung, heart, dorsal root ganglia, and body wall (Cserjesi et al. 1995; Schweitzer et al. 2001; Pryce et al. 2007). In the axial and appendicular skeleton, the $S c x-G F P$ transgenic reporter labels tendon and ligament progenitor cells and all differentiated cell types within these connective tissues (Pryce et al. 2007). We hypothesized that $S c x-G F P$ would serve as a useful marker of middle ear tendon progenitors and permit analysis of middle ear tendon formation. We show that $S c x-G F P$ is a highly specific marker for both tensor tympani and stapedius tendon progenitors, middle ear ligament progenitors, and differentiated tenocytes in the mature tendon. Contrary to expectation, $S c x$ was also expressed in interdental cells, sensory hair cells, and supporting cells in the embryonic inner ear suggesting a unique role in cochlear formation or function.

To gain insight into the putative functions of $S c x$ in the middle and inner ears, we analyzed the $S c x-n u l l$ mouse mutant that has a targeted deletion of exon 1 (Murchison et al. 2007). Scx-null mice are viable but have severe locomotor deficits, postural weakness, and inability to move the tail. In the axial and appendicular skeleton, Scx-nulls display a wide range of tendon phenotypes from incomplete differentiation and shortening of some tendons to the complete absence of other tendons (Murchison et al. 2007). The tensor tympani and stapedius tendons are dramatically shorter in $S c x$ nulls consistent with failed tendon progenitor incorporation into the elongating tendons. While no gross alterations in cochlear morphogenesis or patterning are present in Scx-null mice, $\sim 50 \%$ of nulls demonstrate elevated auditory thresholds. We show that middle ear infection in Scx-nulls accounts for the elevated auditory thresholds in affected mutant mice. We discuss the role of $S c x$ in tensor tympani and stapedius tendon formation and speculate about $S c x$-mediated mechanisms for the pathogenesis of otitis media in Scx-nulls. We conclude that $S c x$ mouse models will be useful tools to advance developmental studies of middle ear tendons and associated musculature and increase our understanding of the biomechanical function of the mature middle ear.

\section{MATERIALS AND METHODS}

\section{Mice}

Male $S c x$ transgenic reporter mice, $S c x-G F P$, were outcrossed to CD1 females to produce embryonic (timed pregnant breeding) or adult middle and inner ears for analysis. $S c x^{-1-}$ (Scx-null) were generated by intercrossing $S c x^{+/-}$mice which also carried the $S c x-G F P$ allele. The validity of the $S c x-G F P$ reporter expression in the middle and inner ears was confirmed by in situ hybridization with the full-length $S c x$ probe (Brown et al. 1999) in CD1 tissues since this probe also detects the Scx-GFP mRNA (Pryce et al. 2007). The breeding paradigm described above and all mouse procedures and animal husbandry protocols described below were approved by the Oregon Health \& Science University Institutional Animal Care and Use Committee.

\section{Auditory brainstem response testing}

Auditory thresholds were determined by auditory brainstem response (ABR) testing (Mitchell et al. 1996). Adult mice (postnatal day 16-28 [P16-18]) were anesthetized using a ketamine $(0.066 \mathrm{mg} / \mathrm{g}$ body weight)/xylazine $(0.013 \mathrm{mg} / \mathrm{g}$ body weight $)$ cocktail in $0.9 \%$ saline. A speaker was connected to the ear canal using a flexible tube, and three electrodes were inserted under the skin. One recording electrode was placed along the sagittal suture of the skull, and two noise canceling electrodes were placed adjacent to the ipsilateral mandible and the distal portion of the ipsilateral foreleg. Tone bursts at 4, 8, 16, and $32 \mathrm{kHz}$ were presented as stimuli. The duration of each tone burst was $2 \mathrm{~ms}$, and responses were recorded for $12 \mathrm{~ms}$. Responses were then averaged to obtain an event related potentials at each frequency and intensity (Mitchell et al. 1996).

\section{Laser interferometric measurement of sound-induced malleal movement}

To explore the cause of the mild hearing loss revealed by the ABRs, the middle ear function was measured in living Scx-null $(n=2)$ and $S c x-G F P(n=1)$ mice. The sound-induced malleal vibrations were measured at different frequencies and sound levels using a custommodified sensitive laser interferometer.

Animal preparation and surgical procedures were similar to those described in previous studies (Ren 2004; He et al. 2008). The initial anesthesia was induced by subcutaneous injection of ketamine $(100-120 \mathrm{mg} / \mathrm{kg})$. The animal's head was firmly attached to a custom-made 
holder with $x-y-z$ translation and rotation capabilities. A tracheotomy was performed to ensure free natural breathing. Body temperature was maintained at $38 \pm 1{ }^{\circ} \mathrm{C}$ with a heating blanket. The $S c x$-GFPand $S c x-n u l l$ mutants without infection have clear middle ear cavities, and the mucosa shows no congestion or inflammation. In contrast, the bony wall of the auditory bulla in the infected Scx-null mice was thickened and difficult to open surgically. The mucosa inside the bulla and on the surface of the ossicular chain was also thicker and swollen. The middle ear cavity was filled with thick yellowish fluid. Hence, for vibration measurements, the fluid above the head of the malleus was removed. Due to the partial removal of this middle ear fluid, the measured reduction in middle ear function was likely underestimated. Moreover, stapes vibration measurements could not be conducted since additional fluid would need to be removed to gain access to the stapes.

The left auditory bulla was opened surgically, and the head of the malleus was visualized through a surgical microscope. One gold-coated glass bead $(\sim 20 \mu \mathrm{m}$ in diameter) was placed on the surface of malleus. The object beam of a modified heterodyne laser interferometer (OFV 302, Polytec, Inc., Germany) was focused on the bead on the malleus through a long-workingdistance objective. The reflected light with Doppler frequency shift from the vibrating bead was collected by the same objective and sent back to the interferometer. The voltage output of a digital decoder was proportional to the vibration velocity of the bead along the optical axis. The noise floor of the measurement was smaller than a picometer (i.e., one trillionth of a meter).

A custom-written program was used to control hardware (System II, Tucker-Davis Technologies, Gainesville, FL) for signal generation and data acquisition. Tone bursts at different frequencies with 23-ms duration and 1-ms rise/fall time were generated by a $\mathrm{D} / \mathrm{A}$ converter. The signals were sent to a power amplifier through a programmable attenuator and then used to drive a speaker. A sensitive microphone (10 B + Etymotic Research, Inc. Elk Grove Village, IL) was used to measure the sound pressure in the ear canal. The microphone-earphone probe was coupled into the external ear canal to form a closed sound field. The signals from the interferometer decoder were digitized with an A/D converter and averaged 10 to 40 times, depending on the signal level. The magnitude and phase of the vibrations at the stimulus frequency were obtained by use of Fourier transformation. The vibrations were measured at sound levels of 60, 70, and $80 \mathrm{~dB}$ SPL and at frequencies from 0.25 to $25.00 \mathrm{kHz}$ in $0.25 \mathrm{kHz}$ steps. The displacement of the malleus vibration $(D)$ in nanometers was obtained from recorded vibration velocity $(V)$ in micrometers per second according to $D=$ $V /(2 \pi f) \times 1,000$, where $f$ is frequency $(\mathrm{Hz})$.
Middle ear dissection and histological preparation

Adult (P16-28) Scx-null mice were fixed by transcardial perfusion with $4 \%$ paraformaldehyde (PFA) in phosphate-buffered saline (PBS; $137 \mathrm{mM} \mathrm{NaCl} ; 2.7 \mathrm{mM} \mathrm{KCl}$; $9.9 \mathrm{mM} \mathrm{Na}_{2} \mathrm{HPO}_{4}, 2 \mathrm{mM} \mathrm{KH}_{2} \mathrm{PO}_{4}, \mathrm{pH}=7.4$ ) and hemisected crania were immersion fixed overnight. The middle ears were dissected under a Leica MZFLIII stereofluorescence dissecting microscope. Skin, muscle, and connective tissue overlying the temporal bone were removed with forceps and a scalpel, and the tympanic membrane was excised with a tungsten wire hook. The middle ear cavity was further exposed by chipping away portions of the bulla with \#5 Inox forceps (Fine Science Tools) filed to $\sim 30^{\circ}$ wedge-shaped point. Adult (P1628) Scx-GFP mice were immersion fixed in $4 \%$ PFA overnight, and then dissected as described above. The Scx-GFP reporter is extremely intense (Pryce et al. 2007), allowing simultaneous bright field (halogen) and epifluorescence $(\mathrm{BF} / \mathrm{EPI})$ illumination to record precise anatomical localization of GFP expression in whole mount middle ears without the need to merge separate bright field and epifluorescence images. BF/EPI-illuminated $S c x-n u l l$ and $S c x$-GFP middle ears were visualized with the Leica MZFLIII stereofluorescence dissecting microscope and imaged with a Retiga 1300 CCD camera.

\section{Plastic thin sections of the adult middle ear}

The tympanic membrane was punctured to facilitate dehydration and infiltration of the middle ear. Opening the middle ear cavity secures optimal histology but, in the case of infected Scx-nulls, displaced some of the cellular infiltrate and middle ear fluid. Whole, fixed $S c x$ null temporal bones were dehydrated in a graded ethanol series $(70 \%, 95 \%, 100 \%$ ethanol for $1 \mathrm{~h}$ each) and vacuum infiltrated $(\sim 25 \mathrm{mmHg})$ with $100 \%$ ethanol/unpolymerized glycomethacrylate (GMA; 1:1 by volume) overnight and $100 \%$ unpolymerized GMA overnight at $22^{\circ} \mathrm{C}$. The tissue was placed in an embedding mold with polymerizing GMA and allowed to cure for $24 \mathrm{~h}$. The tissue block was cut into $5 \mu \mathrm{m}$ sections using a Sorvall JB-4 microtome and mounted on glass slides using Protocol Mounting Medium (Fisher Scientific). Sections were stained with methylene blue/basic fuchsin and visualized with a Leica DM2500 transmitted light/epifluorescence microscope and imaged with a Leica DFC 420C CCD camera.

\section{Cryostat sections}

The whole head of E14.5, E16.5, and E18.5 Scx-GFP embryos were cryoprotected in graded sucrose/PBS (10\%, 20\%, 30\% until the tissue had sunk), embedded in Tissue Freezing Medium (Triangle Biomedical 
Sciences, Durham, NC) and serially sectioned at $12 \mu \mathrm{m}$ in the coronal plane. Slides were mounted in VectaShield (Vector Laboratories, Burlingame, CA). Tissue sections were visualized using a Leica DM2500 transmitted light/epifluorescence microscope and imaged with a Leica DFC 420C CCD camera or the Olympus FV1000 laser-scanning confocal microscope.

\section{Immunohistochemistry}

Scx-GFP embryos at E18.5 were immersion fixed in $4 \%$ PFA in PBS overnight at $4{ }^{\circ} \mathrm{C}$ with gentle agitation. For cryostat sections, the entire head was cryoprotected, embedded, and serially sectioned as described above. For Scx-GFP and Scx-null whole mounts, the cochlea was dissected free of the cartilaginous capsule, and the lateral wall was removed. For tensor tympani and stapedius tendon whole mounts, the malleus and stapes, respectively, were dissected from Scx-GFP and Scx-null adult mice being careful to include a portion of the inserting muscle. The arms of the malleus were trimmed to facilitate orientation for confocal microscopic analyses. All tissues were permeabilized and blocked in $0.2 \%$ saponin in blocking solution (PBS containing $1 \%$ bovine serum albumin and $3 \%$ serum from the species in which the secondary antibody was generated). Antisera against the hair cell marker, Myosin7a, and the muscle marker, Myosin 32 (Myo32), were applied in blocking solution overnight at $4^{\circ} \mathrm{C}$ with gentle agitation. Alexa Fluor conjugated secondary antibodies were applied in blocking solution for $2 \mathrm{~h}$ at $22^{\circ} \mathrm{C}$. Phalloidin-Alexa Fluor conjugates were applied in PBS for $30 \mathrm{~min}$ at $22^{\circ} \mathrm{C}$. Sectioned cochleae and whole mount cochleae were mounted in VectaShield prior to epifluorescence or confocal analysis. Antibodies, labeling reagents, and their dilutions: Myosin 7a (dilution 1/100; 25-6790, Proteus Biosciences Inc, Ramona, CA); Myo32 (dilution 1/1,000; M4276, Sigma); Alexa 568-conjugated goat anti-rabbit (dilution 1/300; A11036, Molecular Probes, Eugene, OR); Alexa 647-conjugated goat anti-rabbit (dilution 1/300;A21245, Molecular Probes); Alexa 660-conjugated phalloidin (dilution 1/150; A22285, Molecular Probes); Alexa 568-conjugated phalloidin (dilution 1/150; A12380, Molecular Probes).

\section{In situ hybridization}

CD1 embryos at E14.5, E16.5, and E18.5 were used for in situ hybridization according to protocols found on the Tabin Laboratory web pages: http://genepath. med.harvard.edu/ cepko/protocol/insituprotocol. pdf. The following probes were used: the $S c x$ fulllength probe (Brown et al. 1999) for tendon and ligament progenitors and differentiated cells within these connective tissues; the Atonal homolog 1 probe (Helms and Johnson 1998) for sensory hair cells; and the Acan probe for chondrocytes (Sandell et al. 1991).

\section{Statistical analyses}

While the $S c x$-GFP mice had normal ABR thresholds, $\sim 50 \%$ of Scx-null mice had elevated thresholds. We initially conducted parametric analyses to gain insight into statistically significant relationships. Pearson correlations indicate that otitis media is a better predictor of elevated average ABR threshold $(N=9$ Scx-nulls [five ears without otitis media, four with otitis media] and $10 S c x$-GFP ears, $r=0.85, p<0.0001)$ than is GFP or null status (10 Scx-GFP ears and 13 Scx-null ears, $r=0.58, p=0.004)$. Moreover, $t$ tests indicate that there is a more significant difference between the average ABR thresholds of animals with otitis media versus those with clear middle ears $(d f=7, t=5.114, p=0.001)$ than there is between the average ABR thresholds of $S c x-G F P$ versus $S c x-n u l l$ mice $(d f=21, t=3.267, p=0.004)$. These statistical differences generally held true for the individual frequencies tested as well as for averaged $\mathrm{ABR}$ responses across frequencies. These results suggest that it is probably not GFP or null status that affects ABR threshold but rather the middle ear infection that is associated with null status. The laser interferometric analysis of Scx-null mice with otitis media was deployed to quantitatively validate this association. Hence, the average ABR thresholds are presented in Figure 2 as mean $\mathrm{dB}$ SPL \pm standard deviation and compared by $t$ test to identify the statistically significant relationships. In addition, the probability values of the $t$ tests comparing average ABR thresholds of Scx-null mice with otitis media to Scx-null mice without otitis media at each frequency tested are presented in Figure 2. $T$ test probability relationships are presented for all other comparisons, and $p$ values are listed in the legend.

\section{RESULTS}

Scx is expressed in sensory and nonsensory cells in the organ of Corti

$S c x$ is a member of the small Twist subfamily of bHLH transcription factors (Franco et al. 2010). Cell fate specification and patterning in the mammalian cochlea are influenced by the balance of bHLH transcription factor activity in otic epithelial progenitor cells (Kelley et al. 2009). We, therefore, evaluated the expression of $S c x$ in the E18.5 mouse organ of Corti using the Scx-GFP transgenic reporter mouse in which green fluorescent protein expression is driven by regulatory sequences of the Scx gene (Pryce et al. 2007). We detected robust GFP expression in cryostat sections of the E18.5 mouse cochlea (Fig. 1A-D). GFP co-localized with myosin $7 \mathrm{a}$ 
(Myo7a) in inner hair cells and was weakly expressed in outer hair cells (Fig. 1A, B, D). GFP was also expressed in a superficial layer of cells in the spiral limbus with distinct apical associations consistent with interdental cell morphology (Fig. 1A, G, J, arrows). In addition, GFP was present in cuboidal supporting cells on the strial side of the organ of Corti consistent with Claudius cell identity (Fig. 1A, arrowhead, D). Scx-GFP expression in the E18.5 cochlea largely agrees with $S c x$ mRNA expression, with the notable exception of outer hair cells which have weak $S c x$-GFP expression but robust $S c x$ mRNA expression (Fig. 1E, F, asterisks). Confocal analysis of P1 Scx-GFP cochlear whole mounts (Fig. 1G-J) further confirmed robust $S c x$ expression in
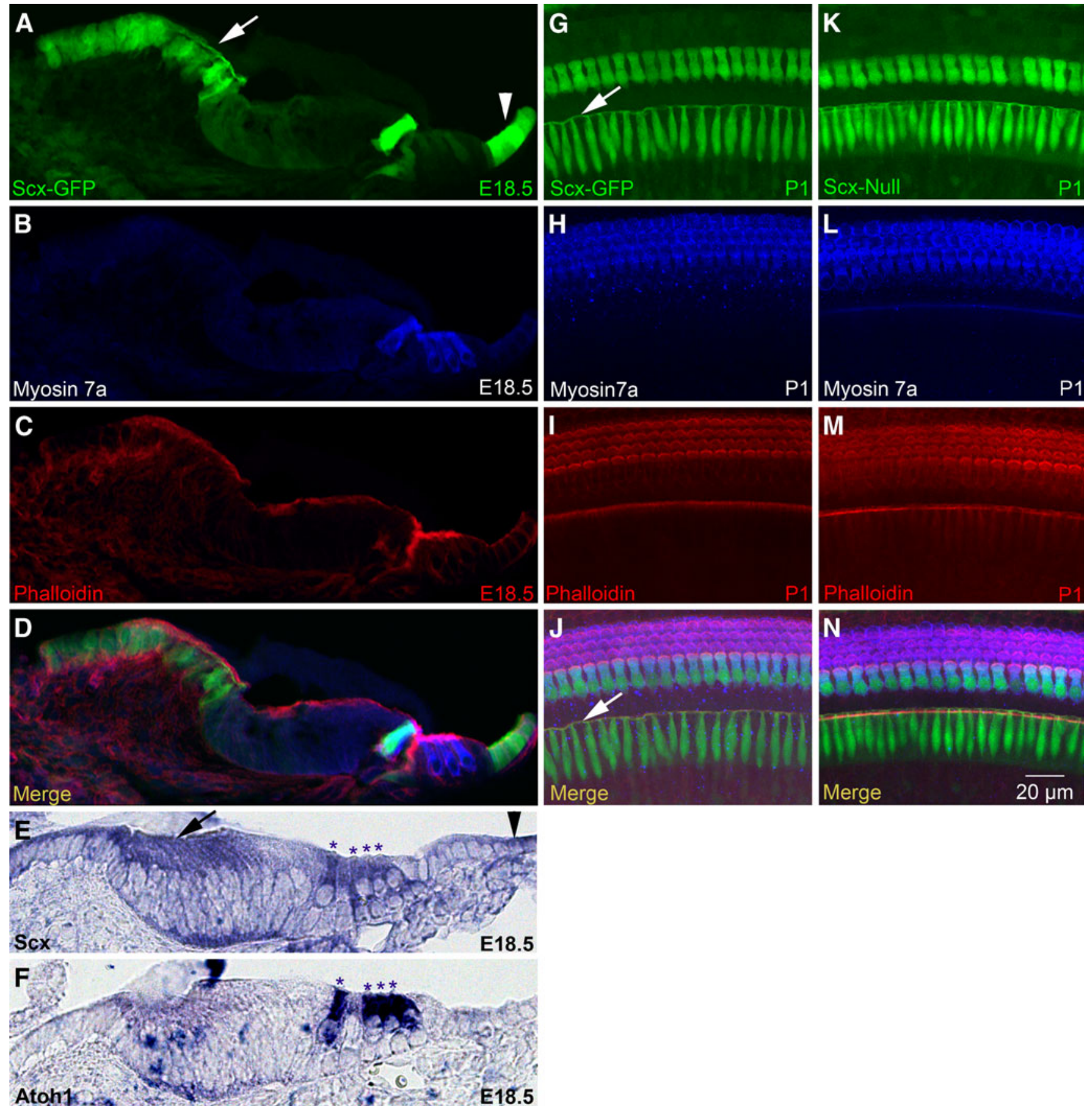

FIG. 1. SCX is expressed in interdental cells, sensory hair cells, and Claudius cells. A-D Confocal microscopy of a cryostat section from the E18.5 SCX-GFP organ of Corti labeled with myosin 7a (B) and phalloidin (C). Panels A-C are merged in D. E, F In situ hybridization for Scx (E) and Atoh1 (F) mRNA in cryostat sections of the E18.5 wild-type organ of Corti. Asterisks indicate apices of inner and outer hair cells. G-N Confocal microscopy of whole mount SCX-GFP G-J and $S C x-n u l l \mathbf{K}-\mathbf{N}$ organs of Corti labeled with myosin 7 a $\mathbf{H}, \mathbf{L}$ and phalloidin $\mathbf{I}, \mathbf{M}$. Panels $\mathbf{G}-\mathbf{I}$ are merged in $\mathbf{J}$, and panels $\mathbf{K}-\mathbf{M}$ are merged in $\mathbf{N}$. Arrows indicate interdental cells and the arrowheads indicate Claudius cells. The cryostat section in $\mathbf{A}-\mathbf{D}$ is from a more basal location in the cochlea than the sections in $\mathbf{E}$ and $\mathbf{F}$. Scale bar in $\mathbf{N}$ applies to all panels. 
inner hair cells (Fig. 1G-J) and interdental cells (Fig. 1G, J, arrows).

$S c x$ is not required for gross morphogenesis or patterning of the organ of Corti

To determine if $S c x$ is required for cochlear development, we evaluated the $S c x$-null mouse bearing the
$S c x$-GFP reporter transgene. GFP expression in the $S c x$ null mouse thus identifies cells that would normally express $S c x$, though no functional $S c x$ gene product is produced in the mutant background. Confocal analysis of E18.5 Scx-null cochlear whole mounts (Fig. 1K-N) demonstrated GFP in inner hair cells and interdental cells $($ Fig. $1 \mathrm{~K}, \mathrm{~N}$ ) in a pattern indistinguishable from $S c x$ GFP expression (compare Fig. 1K-N to G-J). Moreover,
A

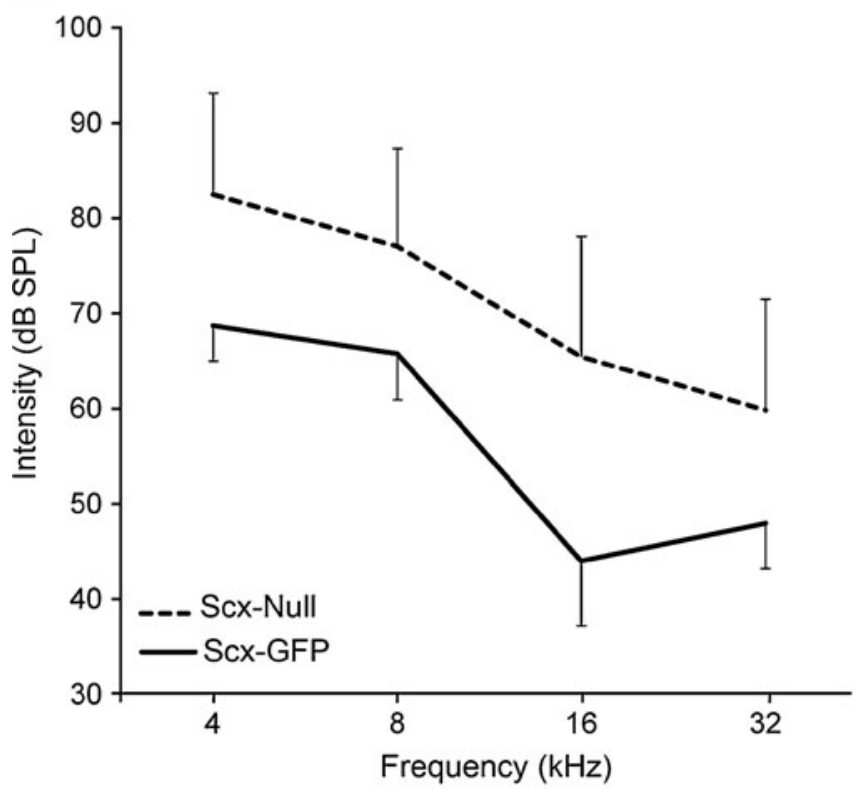

B

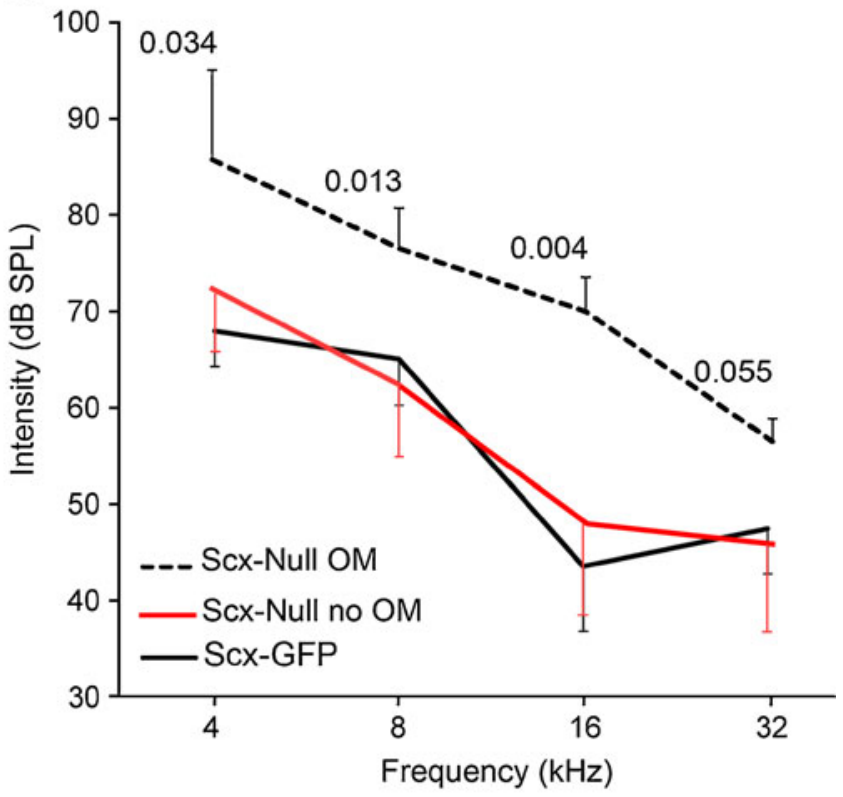

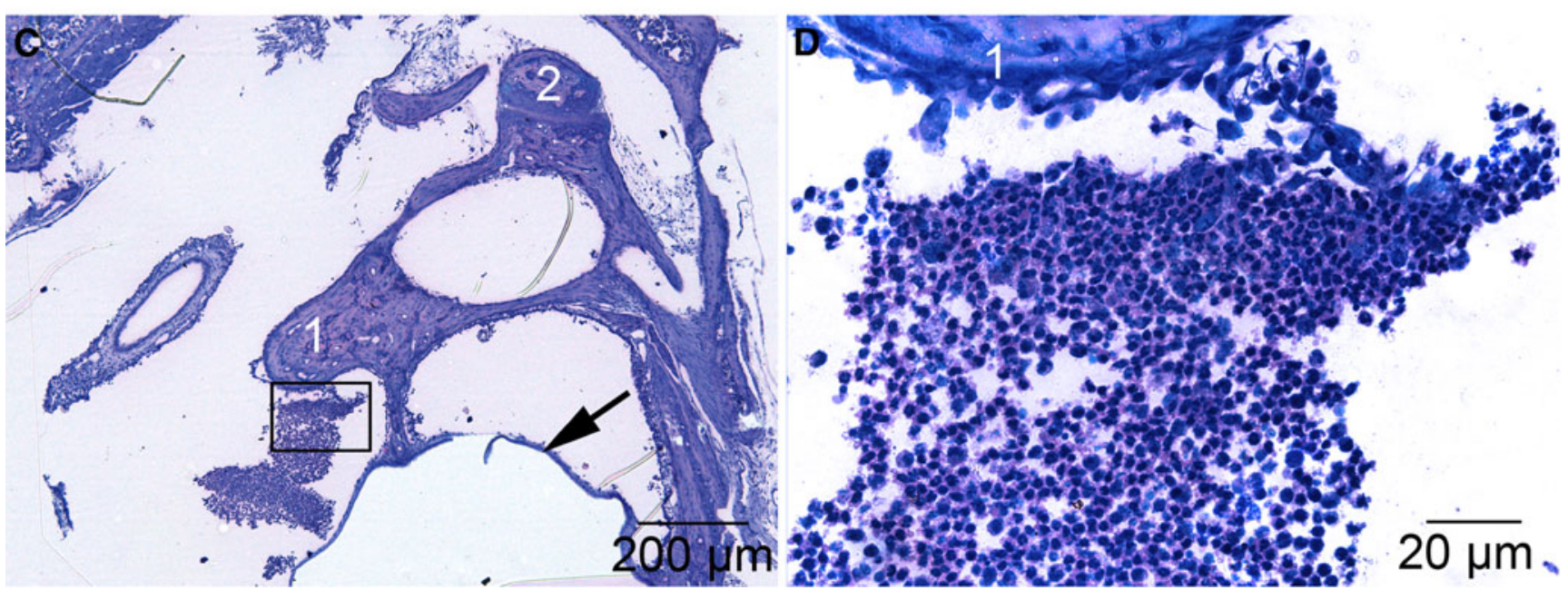

FIG. 2. Middle ear infection correlates with elevated hearing thresholds in affected $S c x$-null mice. A The average auditory brainstem response thresholds in sound pressure level (dB SPL) as means \pm standard deviation (SD) are plotted for $S_{C X}$-null ( $n=26$ ears; 13 mice) and $S_{C X}-G F P(n=20$ ears; 10 mice) mice at 4, 8, 16, and $32 \mathrm{kHz}$. Auditory thresholds in $S C X$-null mice are statistically significantly elevated at all four frequencies tested $(t>2.02 ; p<0.001)$. B The average auditory brainstem response thresholds (dB SPL $\pm \mathrm{SD}$ ) are plotted for $S_{C X}$-null mice with otitis media (OM; dashed line, $n=4$ ears from four mice) versus $S c x$-null mice without otitis media (red line; $n=5$ ears from five mice) at 4, 8, 16, and $32 \mathrm{kHz}$. The average ABR thresholds of $S C x$-null mice with otitis media are statistically significantly elevated at three of the four frequencies tested ( $t$ test, $p$ values indicated on the graph) compared to $S_{C X}$-null mice without otitis media. The ABR thresholds of $S_{C X}$-null mice without otitis media (B; red line) are not statistically significantly different from $S_{C X}$-GFP mice (B, black line; data from $\left.\mathbf{A}\right)$ at all frequencies tested $(t>2.07, p>0.12)$. C, D Methylene blue/basic fuchsin-stained plastic thin sections of a representative $S_{C X}$-null middle ear that presented with white, viscous fluid behind the tympanic membrane upon postmortem analysis. The boxed area in $\mathbf{C}$ is shown at higher magnification in (D). The methylene blue-stained cells between the malleus (1) and tympanic membrane (arrow) have small nuclei with scant cytoplasm consistent with infiltrating lymphocytes. Weakly methylene blue-stained acellular debris is associated with the incus (2). 

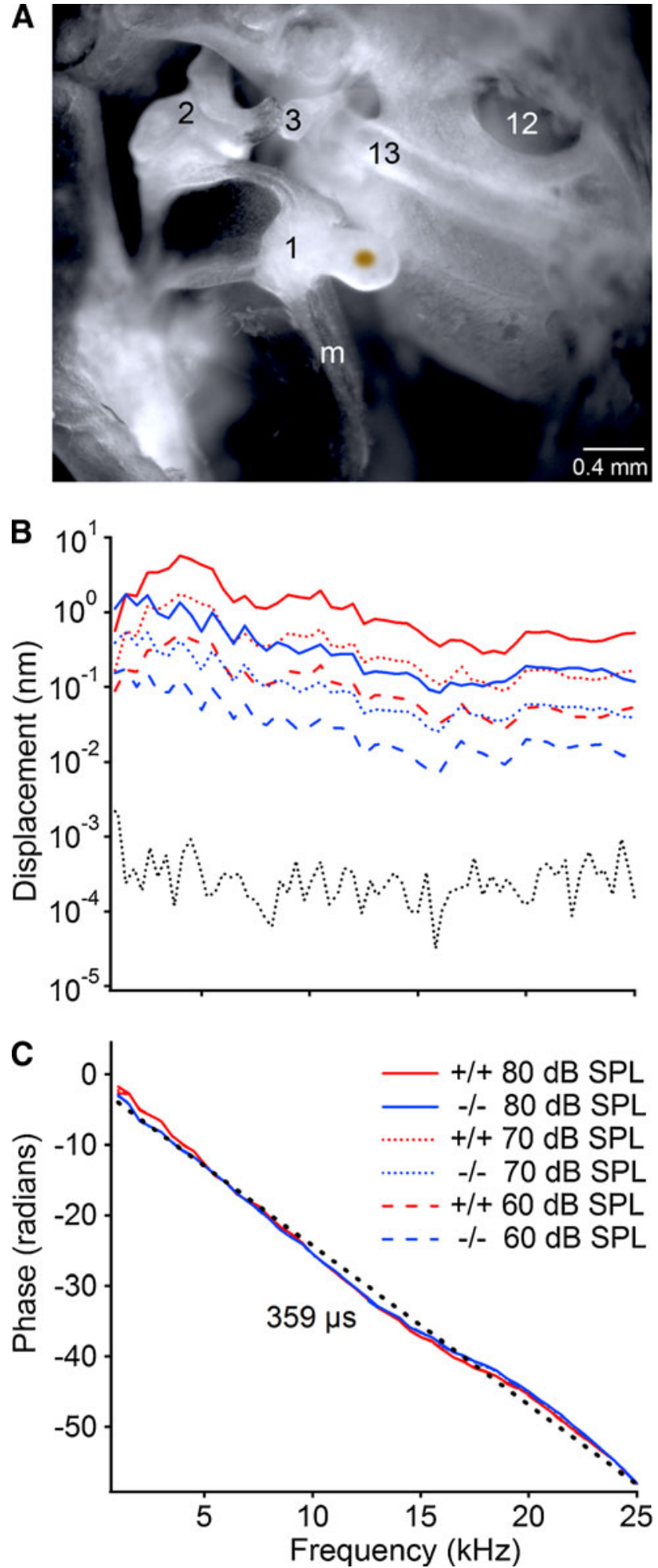

the $S c x$-null organ of Corti was properly patterned with one row of inner and three rows of outer hair cells which displayed apical, phalloidin-positive (phalloidin ${ }^{+}$) stereociliary bundles, and normal planar polarity (Fig. 1L-N). These data suggest that $S c x$ is not required for gross morphogenesis and patterning of the organ of Corti.
FIG. 3. Ossicle movements are dampened in Scx-null mice with otitis media. A Brightfield image of the clear, adult mouse middle ear demonstrating placement of the gold-coated glass bead on the medial face of the malleal head for laser interferometric quantitation of malleal movement. Inflammatory cells and fluid were partially cleared from the middle ear cavity to permit placement of the bead in SCX-null mice with otitis media, suggesting that the laser interferometric estimates are likely an underestimation of the decrease in malleal vibration. 1 malleus; 2 incus; 3 stapes; 13 stapedial artery; 12 round window; $m$ manubrium of the malleus. B The vibration magnitude of the malleus in nanometers is plotted as a function of frequency in $\mathrm{kHz}$ at 60, 70, and $80 \mathrm{~dB}$ SPL. Color key applies to panels B and $\mathbf{C} ;+/+$ is $S C X-G F P ;-/-$ is $S C X-n u l l$. Data from a representative $S C X-G F P$ and $S C X$-null mouse are shown. The dotted gray line is the measured noise floor for the laser interferometric measurements. C The phase in radians is plotted as a function of frequency at $80 \mathrm{~dB}$ SPL. The slope of the linear regression line (dotted black line) indicates the group delay $(\sim 359 \mu \mathrm{s})$. The solid red and blue lines are the observed phase/frequency curves from the SCX-GFP and SCX-null mice at $80 \mathrm{~dB}$ SPL.

\section{Hearing loss in $S c x$ null mice is associated with middle ear infection}

Molecular defects in interdental cells, hair cells, or supporting cells in the organ of Corti are associated with auditory dysfunction (Zwaenepoel et al. 2002; Zhu and Zhao 2010). Since $S c x$ is expressed in all of these cell types, we measured auditory thresholds in Scx-null and control Scx-GFP mice. The average ABR thresholds ( \pm standard deviation [SD]) of Scx-null mice ( $n=26$ ears from 13 mice) are statistically significantly elevated $(t>$ $2.02, p<0.001)$ at $4,8,16$, and $32 \mathrm{kHz}$ compared to $S c x$ GFP mice ( $n=20$ ears from 10 mice; Fig. 2A).

We were surprised to find, however, that the hearing loss in Scx-null mice was heterogeneous with some mice presenting elevated thresholds and others with normal thresholds. The ears from nine of the 13 Scx-null mice whose brainstem audiometric responses were included in the ABR average in Figure 2A were separated into two subpopulations postmortem defined by the absence or presence of pathological evidence of middle ear infection. Viscous white or yellow fluid in the middle ear cavity that blurred visual identification of the malleus when viewed laterally (i.e., from the external acoustic meatus; see Fig. 3A) was considered a sign of middle ear infection. Tightly packed clusters of methylene blue-stained lymphocytes were detected in plastic thin sections from the middle ear of a representative Scx-null mouse with pathological signs of middle ear infection (Fig. 2C, D). Lymphocytic infiltration into the middle ear space was not detected in Scx-null mice with visually clear middle ears or in control $S c x$-GFP mice (data not shown). We compared the average ABR thresholds \pm SD of $S c x-n u l l$ mice with middle ear infection $(n=4$ ears from four mice) to $S c x-n u l l$ mice without middle ear infection ( $n=5$ ears from five mice; Fig. 2B). The ABR thresholds of Scx-null mice with infection are statistically significantly elevated at three of the four frequencies 

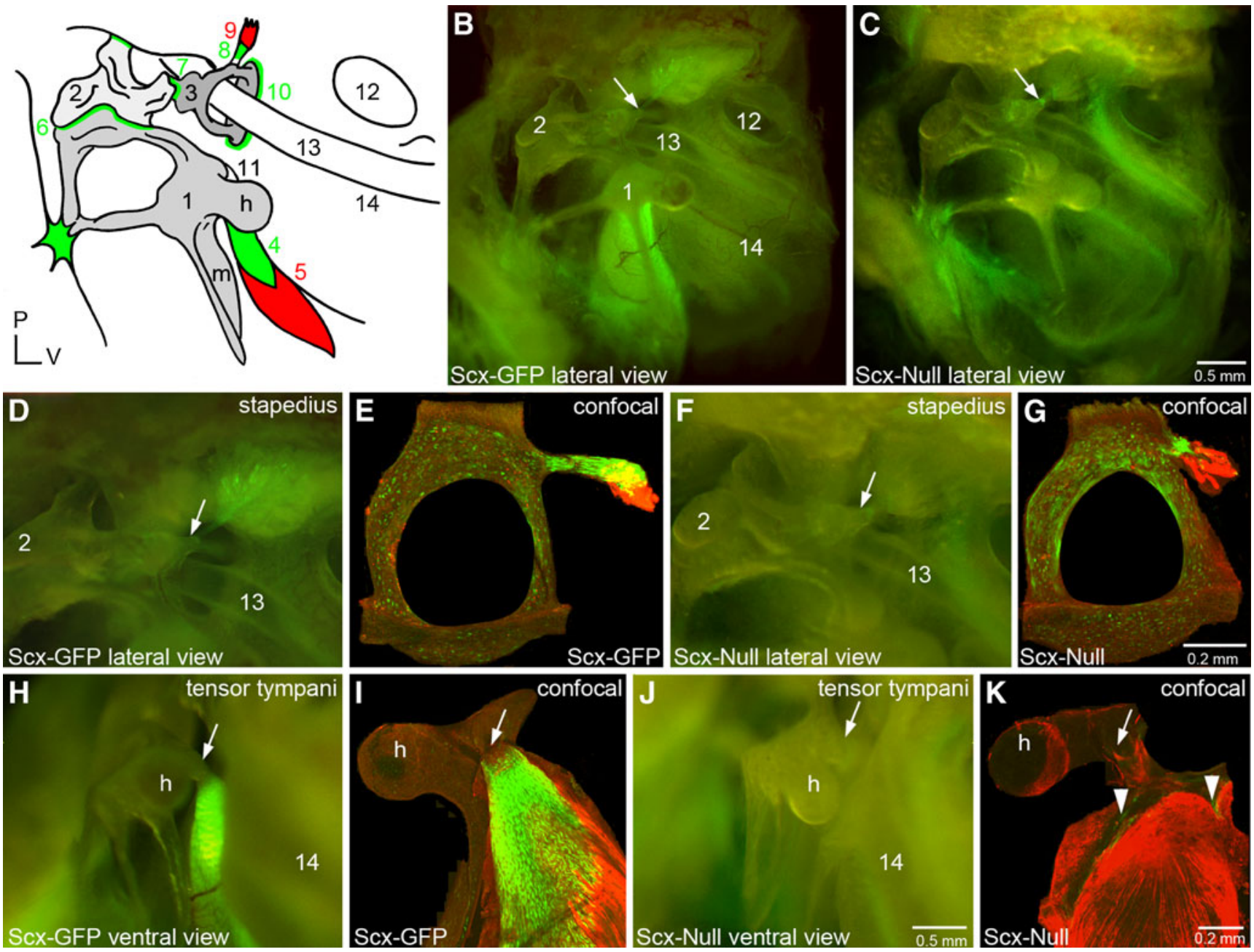

tested ( $t$ test, probability values indicated on the graph in Fig. 2B) compared to $S c x$-null mice without middle ear infection (Fig. 2B, red line, $n=5$ ears). In addition, the ABR thresholds of $S c x-n u l l$ mice without infection are not statistically significantly different from control $S c x-G F P$ mice (Fig. 2B; black line, $n=26$ ears) at all frequencies tested $(t>2.07, p>0.12)$. These data suggest that the hearing loss observed in a subset of Scx-nullmice correlates with middle ear infection or otitis media.

Scx-null mice with otitis media display conductive hearing loss

To estimate the contribution of otitis media to the elevated auditory thresholds in affected Scx-null mice, we measured the mechanical movements of the malleus in response to sound of varying frequency and intensity by laser interferometry (Fig. 3A). The noise floor of the interferometric analysis was in the picometer range (Fig. 3B, dotted gray line), two orders of magnitude below the magnitude of the measured ossicle movements. The group delay $(\sim 359 \mu \mathrm{s})$ measures the time elapsed for the sound stimulus to travel from the speaker to the malleus and this value is similar for both $S c x-G F P(+/+)$ and $S c x-n u l l$ $(-/-)$ mice at all frequencies tested (Fig. 3C). The vibration magnitudes in $S c x-n u l l$ mice with otitis media (Fig. 3B, blue lines) are $\sim 5 \mathrm{~nm}(\sim 10 \mathrm{~dB})$ below those of the $S c x-G F P$ mouse (Fig. 3B, red lines) at corresponding sound pressure levels indicating increased impedance of the middle ear. Malleus movement decreased slightly with frequency at all sound intensities tested. Thus, the $\sim 10 \mathrm{~dB}$ decrease of sound transmission due to middle ear infection in affected Scx-null mice can account for the elevated ABR thresholds observed (Fig. 2). This observation, along with normal ABR thresholds in Scx-null mice without infection, strongly suggests that the hearing loss in affected $S c x-n u l l$ mice is conductive in nature and caused by the cellular infiltrate and viscous fluid associated with otitis media.

\section{Scx-GFP is a robust reporter for middle ear tendons}

The acoustic reflex in mammals reduces transmission of loud sound to the cochlea thereby protecting the sensory epithelium from damage (Mukerji et al. 2010). The reflex is mediated by contraction of the stapedius and tensor tympani muscles which insert into the stapes 
FIG. 4. $S_{C X}$ is required for middle ear tendon differentiation. A The mammalian middle ear consists of three delicate bones and associated ligaments and tendons. The manubrium $(m)$ of the malleus (1) is inserted into the tympanic membrane or ear drum (see also Fig. 2C, arrow). The incus (2) articulates with the malleus and the stapes (3) at the malleoincudal (6) and incudostapedial (7) joints, respectively, where ligaments provide structural integrity. The footplate of the stapes rests on the oval window of the cochlea where it is stabilized by the annular ligament ( 10 ). The tensor tympani muscle (5) inserts into a tubercle (11) adjacent to the head ( $h$ ) of the malleus through the tensor tympani tendon (4). The stapedius muscle (9) inserts into a tubercle on the posterior arch of the stapes through the stapedial tendon (8). The round window of the cochlea (12), the stapedial artery (13), and the bony labyrinth at the level of the middle turn of the cochlea (14) are prominent anatomical landmarks in this lateral view. All middle ear tendons and ligaments express Scx and green shading schematically summarizes Scx-GFP expression. A patch of ScX-GFP-expressing tissue is localized to the arms of the malleus where they associate with the dorsal wall of the middle ear cavity. A small focus of $S_{C X}$-GFP-expressing tissue is also present between an arm of the incus and the posterior wall of the middle ear cavity. $P$ posterior; $V$ ventral. These numerical and alphabetic labels are used in subsequent panels $(\mathbf{B}, \mathbf{C})$ Lateral view of the $S_{C X}-G F P(\mathbf{B})$ and $S_{C X}-n u l l(\mathbf{C})$ middle ears imaged in combination bright field/epifluorescence (BF/EPI) mode (see Materials and methods). The stapedial tendon insertion point (arrow) on the tubercle of the posterior arch of the stapes is indicated. The tensor tympani tendon located behind the malleus (1) robustly expresses $S c x-G F P$ in wild type (B) but not the null (C) middle ear. SCX-GFP is not detectable in the middle ear mucosa. SCX-GFP expression in the cochlea is detectable through the bony labyrinth in the wild type (14) and null inner ears. Scale bar in (C) applies to (B). (D, E) The stapedial tendon insertion into the tubercle on the posterior arch of the stapes in the SCX-GFP middle ear imaged by BF/EPI (D) and laser confocal (E) microscopy. The tenocytes in the stapes tendon robustly express GFP that is detectable in the whole mount image as a feathered array of green fluorescence terminating at the posterior arch of the stapes (D, arrow). The excised stapes was dissected from its associations with the oval window and incus prior to immunohistochemical detection of Myo32 and confocal analysis. The stapes tendon insertion into the tubercle and its association with a fragment of the Myo32 $2^{+}$stapedius muscle is shown in (E). The posterior arch of the stapes in (E) was fractured during excision or tissue processing $(\mathbf{F}, \mathbf{G})$ SCX-GFP expression is weakly detectable in the BF/EPI (F) and laser confocal $(\mathbf{G})$ images of the stapes of $S_{C X}-n u l l$ mice. The stapedius is inserted into the stapes by a GFP ${ }^{+}$tendon $(\mathbf{F}$, arrow and $\mathbf{G}$; see also $\mathbf{C}$, arrow). The stapedial tendon in $S c x-n u l l$ mice is dramatically shorter than the tendon in the wild type $S_{C X}$-GFP middle ear (compare GFP ${ }^{+}$tendon in $\mathbf{E}$ and $\mathbf{G}$ ). The foreshortened stapedius tendon is associated with a fragment of the Myo32 $2^{+}$stapedius muscle in the $S_{C X}$-null middle ear $(\mathbf{G})$. Scale bar in $\mathbf{G}$ applies to $(\mathbf{E})$. ( $\mathbf{H}$, I) The tensor tympani tendon insertion into the tubercle of the malleus in the $S_{C X}$-GFP middle ear imaged by BF/EPI $(\mathbf{H})$ and laser confocal (I) microscopy. The tenocytes in the tensor tympani robustly express $S_{C X}-G F P(\mathbf{H})$, and it is this intense expression that is detectable behind the malleus in the lateral view of the $S_{C X}-G F P$ middle ear in panel $\mathbf{B}$. The matrix that anchors the tendon to the tubercle is GFP negative $(\mathbf{H}$, arrow). The excised malleus was dissected from its associations with the ear drum and incus, and the bony arms were trimmed prior to immunohistochemical detection of Myo32 and confocal analysis. Myo32 expression in the tensor tympani (I; red) demonstrates the extensive association of the GFP ${ }^{+}$tendon with the muscle and the absence of GFP expression proximal to the insertion point on the tubercle (I, arrow). (J, K) ScX-GFP expression is weakly detectable in BF/EPI (F) and laser confocal $(\mathbf{G})$ images of the malleus in $S c x-n u l l$ mice. Confocal analysis detects a small complement of diffusely arrayed, weakly GFP ${ }^{+}$ tenocytes (K, arrowheads) associated with the Myo32 ${ }^{+}$tensor tympani. The tensor tympani inserts into the malleus by a foreshortened, stubby cord of matrix that is GFP negative (J, arrow and Fig. 6). The reduced expression of $S_{c x}$-GFP in the $S_{c x}$-null tensor tympani tendon accounts for the inability to detect GFP expression behind the malleus in the BF/EPI image (J) and in the lateral view of the $S_{C X}$-null middle ear (C). Scale bar in (J) applies to (D, F, H). Scale bar in (K) applies to (I).

and malleus by their respective tendons (Fig. 4A). Scx was previously shown to be expressed in differentiated tendon cells called tenocytes in all tendons of the axial and appendicular skeleton (Pryce et al. 2007). We, therefore, analyzed the expression of $S c x$ in the mature middle ear of $S c x-G F P$ mice to determine if $S c x$ is also a marker for middle ear tendons. GFP expression is present near the stapes in the $S c x$-GFP middle ear in lateral view (Fig. 4B, arrow). The GFP expression appears in a feathered distribution that coalesces onto the posterior arch of the stapes in ventral view of the intact middle ear (Fig. 4D, arrow). Confocal analysis of the stapes excised from the middle ear and immunostained with the muscle marker myosin 32 (Myo32) confirms $S c x$ expression in the stapedius tendon which overlaps with the stapedius muscle (Fig. 4E).

Intense GFP expression is also present behind the body of the malleus in an arc that traces the angle of the manubrium in the $S c x-G F P$ middle ear in lateral view (Fig. 4B). The GFP expression localizes to the surface of the tensor tympani muscle in ventral view of the intact middle ear (Fig. 4H). Confocal analysis of the excised malleus confirms Scx-GFP expression in the Myo32-negative tensor tympani tendon (Fig. 4I). GFP is absent from the terminal portion of the tendon at the insertion into the malleus (Fig. 4H, I, arrows). In summary, the expression data suggest that Scx-GFP is a robust reporter for middle ear tendons.

\section{$S c x$-GFP accurately reports $S c x$ mRNA expression in tenocytes}

To determine whether $S c x-G F P$ accurately reports $S c x$ mRNA expression in tenocytes of tendons, we performed in situ hybridization in cryostat sections of the middle ear at E18.5 when the tendons are inserted into their target ossicles and display advanced morphogenetic development. Since the full-length $S c x$ mRNA probe also detects the $S c x-G F P$ mRNA (Pryce et al. 2007), we performed our analyses in wild-type mice. We then compared mRNA expression in wild-type middle ears to that in age-matched $S c x$-GFP middle ears. The stapedius tendon and tensor tympani tendon express $S c x$-GFP robustly proximal to their respective insertion sites (Fig. 5G, J) consistent with the whole mount images previously shown. $S c x$-GFP (Fig. 5G, J) and $S c x$ mRNA (Fig. $5 \mathrm{H}, \mathrm{K}$ ) are robustly expressed in tenocytes in both the stapedius tendon (compare Fig. 5G, H, white and black arrows) and the tensor tympani tendon (compare Fig. 5J, K, arrowheads) at E18.5. 

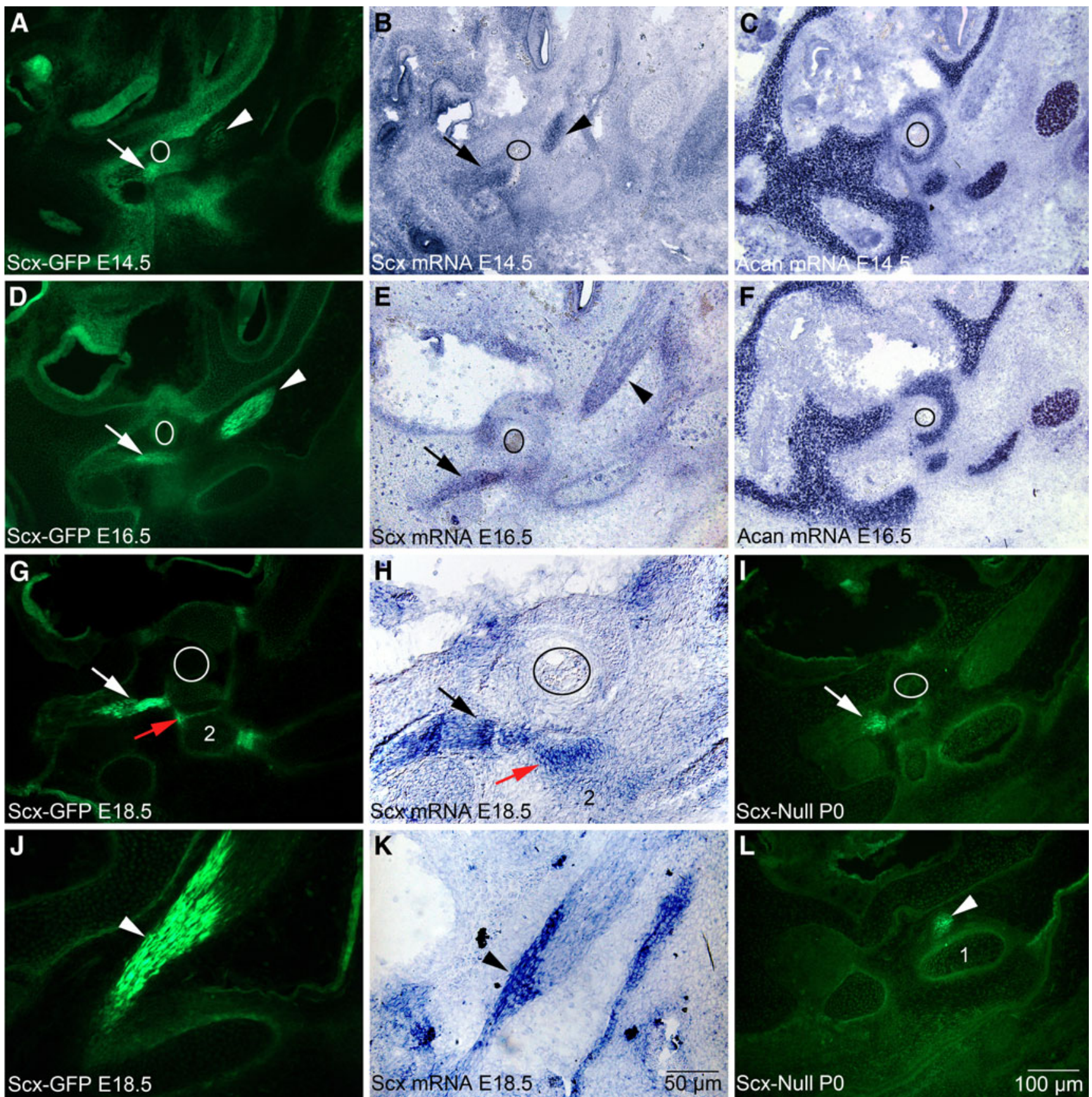

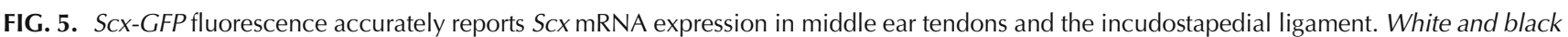
arrows indicate the developing stapedius tendon, and arrowheads indicate the tensor tympani tendon in all panels. A, D, G, J, I, L SCX-GFP expression at E14.5 (A), E16.5 (D), E18.5 (G, J), and P0 (I, L) in cryostat sections of the SCX-GFP middle ear. C, F Acan expression in chondrocytes of the nascent stapes at E14.5 (C) and E16.5 (F) and chondrocytes in the developing cartilaginous capsule of the inner ear. The circle outlines the obturator foramen of the stapes through which the stapedial artery passes. Acan provides an anatomical framework to interpret $S C X-G F P$ and mRNA expression in (A, B, D, E). $\mathbf{H}, \mathbf{K} S C x-m R N A$ expression in the stapedius tendon ( $\mathbf{H}$, black arrow) and tensor tympani tendon (K, arrowhead) at E18.5. The narrow band of $S C X-G F P$ expression (G, red arrow; 2, incus) in the incudostapedial ligament correlates with $S C X$ mRNA expression $(\mathbf{H}$, red arrow). Scale bar in $(\mathbf{K})$ applies to $(\mathbf{H}, \mathbf{J})$; scale bar in $(\mathbf{L})$ applies to all other panels.

$S c x$-GFP accurately reports $S c x$ mRNA expression in tendon progenitors

Scx was also previously shown to be expressed in tendon progenitor cells in all developing tendons of the axial and appendicular skeleton (Schweitzer et al. 2001; Murchison et al. 2007; Pryce et al. 2007). To identify the early stages in the formation of middle ear tendons, we analyzed $S c x$-GFP and $S c x$ mRNA expression at E14.5 and E16.5 when middle ear tendon progenitors 
condense proximal to their bony insertion sites and tenocytes become incorporated into the elongating tendon. The Acan probe (Sandell et al. 1991) was used as a convenient marker for chondrocytes that facilitated identification of the developing ossicles and cartilaginous capsule of the cochlea (Hwang and Wu 2008) (Fig. 5C, F). Tendon progenitors that give rise to the stapedius and tensor tympani tendons are condensed into $\mathrm{GFP}^{+}$clusters in close proximity to their target ossicles at E14.5 (Fig. 5A-C) and begin incorporation into the growing long axis of the tendon by E16.5 (Fig. 5D-F). Scx mRNA expression in differentiating tenocytes closely matches $S c x-G F P$ distribution at E14.5 and E16.5 (compare Fig. 5A, B, D, E). We conclude that $S c x-G F P$ accurately reports $S c x$-mRNA expression in both tendon progenitor cells and differentiated tenocytes of the middle ear.

\section{$S c x$ is required for middle ear tendon formation}

Scx-null mice display a complex range of tendon phenotypes ranging from poorly differentiated tendons to the complete absence of some tendons (Murchison et al. 2007). To determine if $S c x$ is required for middle ear tendon formation, we analyzed the Scx-null mouse carrying the $S c x-G F P$ reporter allele (Murchison et al. 2007). A small focus of GFP expression is present near the head of the stapes in the Scx-null middle ear in lateral view (Fig. 4C, arrow). GFP is weakly detected near the posterior arch of the stapes in the ventral view of the intact middle ear (Fig. 4F, arrow). Confocal analysis of the excised stapes immunostained with Myo32 confirms expression in the foreshortened, $\mathrm{GFP}^{+}$stapedius tendon that associates with a fragment of the Myo32 ${ }^{+}$stapedius muscle (Fig. 4G). In summary, $\mathrm{GFP}^{+}$tenocytes are present in the stapedius tendon in Scx-null mice, the tendon is dramatically shorter than the wild-type tendon, and the stapedius is anchored to the stapes.

In contrast to the stapedius tendon phenotype, GFP expression is absent from the malleal territory in the $S c x$-null middle ear in lateral view (Fig. 4C) and $S c x$-GFP in the basal and middle turns of the cochlea is apparent (Fig. 4C). GFP is only weakly detected above background in the ventral view of the malleal territory (Fig. 4J, arrow). Confocal analysis of the dissected malleus immunostained with Myo32 confirms the absence of organized $S c x$-GFP expression in the tensor tympani territory with only diffuse $\mathrm{GFP}^{+}$puncta associated with the Myo32 ${ }^{+}$tensor tympani muscle (Fig. 4K, arrowheads). To determine if the tensor tympani tendon forms in Scx-null mice, we analyzed the malleus excised from the middle ear with the tensor tympani muscle by combined BF/EPI microscopy (Fig. 6). In bright field, the tensor tympani tendon appears as a residual band of connective tissue that anchors the tensor tympani to the malleus
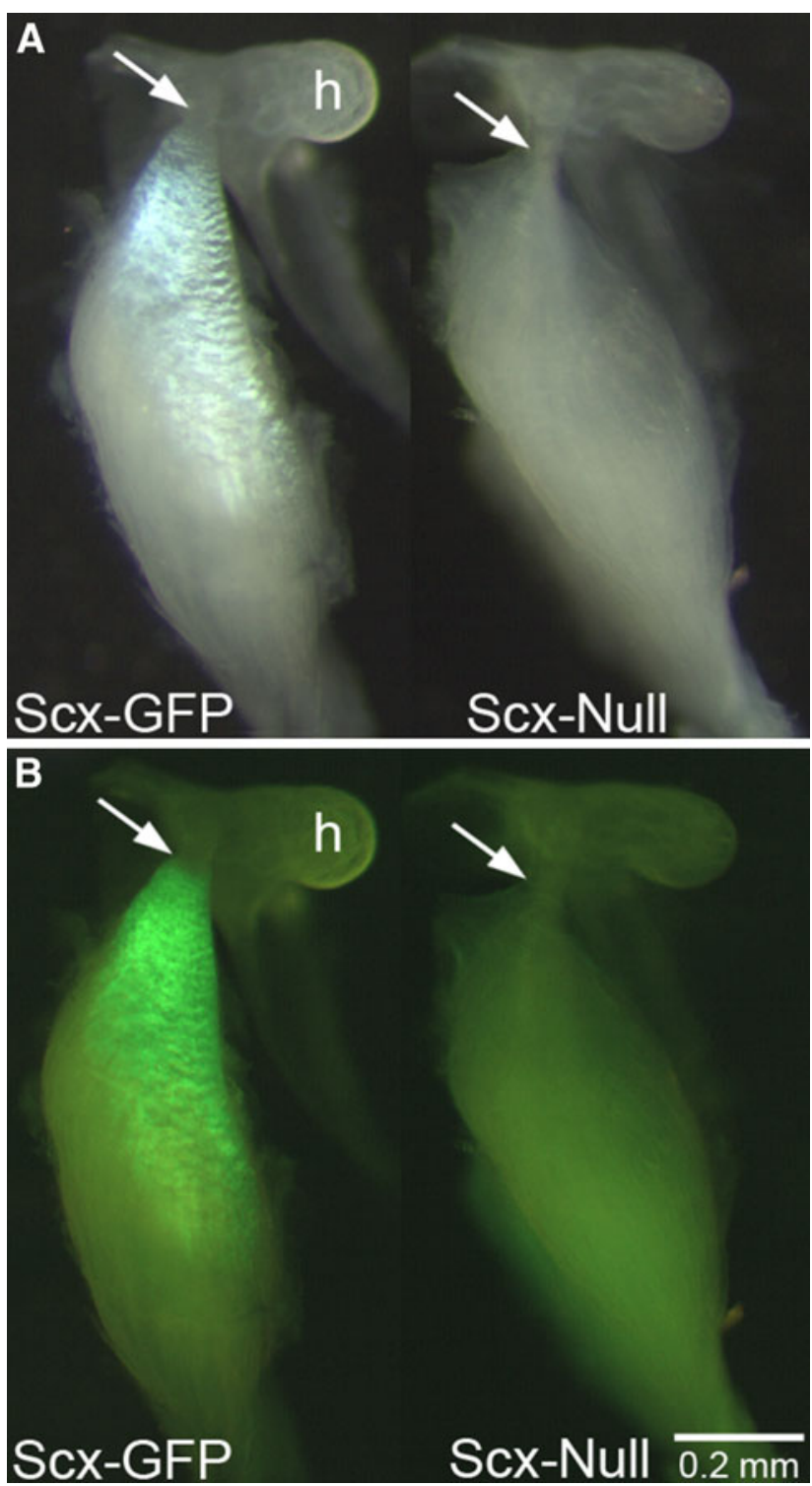

FIG. 6. $S C X$ is required for tensor tympani tendon differentiation. Bright field (A) and BF/EPI (B) images of the SCX-GFP (left) and SCXnull (right) tensor tympani and malleus. (A, left) Collagen 1 in the SCX-GFP tensor tympani tendon reflects bright field illumination creating a pearly white appearance. The tendinous insertion into the malleal tubercle is indicated by the arrow. The $S_{C x}$-null tensor tympani does not possess the collagen 1 reflective surface but the muscle inserts into the malleal tubercle (A, right, arrow). GFP ${ }^{+}$ tenocytes are present throughout the $S_{C X}$-GFP tensor tympani tendon, though the terminal insertion is GFP negative (B, left, arrow). No obvious $\mathrm{GFP}^{+}$tenocytes are detected in the whole mount preparation of the $S c x$-null tensor tympani (B, right; see also Fig. 4, $\mathbf{K}$, arrowheads). Scale bar in (B) applies to (A).

(Fig. 6A, right, arrow). In contrast, the pearly white collagen 1 matrix of the $S c x-G F P$ tensor tympani extends over a large surface area of the muscle (Fig. 6A, left). Epifluorescence microscopy fails to detect GFP expression above background in the $S c x$ null tensor tympani tendon (Fig. 6B, right), while GFP is robustly expressed in the $S c x-G F P$ tensor tympani 
(Fig. 6B, left). In summary, despite the presence of only a few $\mathrm{GFP}^{+}$tenocytes in the tensor tympani tendon in $S c x$-null mice and the severe reduction of the collagen 1 matrix, a residual tendon matrix that anchors the tensor tympani to the malleus is formed in Scx-null mice.

To gain further insight into the Scx-null tendon phenotype, we analyzed cryostat sections of the P0 middle ear, a time point in wild-type mice by which tenocytes are incorporated into the long axis of the stapedius and tensor tympani tendons. In the Scx-null mutants, the $\mathrm{GFP}^{+}$tenocytes in the stapedius and tensor tympani are present at $\mathrm{P} 0$, though they are not patterned in a linear array coincident with the long axis of the tendon (Fig. 5I, L) as they are by E18.5 in Scx-GFP tendons (Fig. 5G, J). The $S c x$-null tenocytes are localized adjacent to their bony insertion sites and produce a sufficiently stable extracellular matrix to anchor muscle to bone (Figs. 4G and 6). The $S c x$-null tendon phenotype is consistent with failed differentiation in which tenocytes are not incorporated into the growing tendon and reduced tendon matrix is produced.

\section{$S c x$ is expressed in middle ear ligaments}

Scx is also a marker for ligaments in the axial and appendicular skeleton (Murchison et al. 2007; Pryce et al. 2007). We investigated whether ligaments in the middle ear also express $S c x$. The middle ear contains three articulating joints associated with three ligaments: the stapes footplate with the oval window (annular ligament); the stapes with the incus (incudostapedial ligament); and the incus with the malleus (malleoincudal ligament; Fig. 4A). We detected $S c x-G F P$ and $S c x$ mRNA expression in the annular ligament (Fig. 7A, B, red arrowheads); in the medial (Fig. 7A, B, white arrowheads) and lateral (Fig. 7D, E) regions of the malleoincudal ligament; and in the incudostapedial ligament (Fig. 5G, H, red arrow). Middle ear ligaments in $S c x$-null mice appear to be intact (Fig. 7C, F), consistent with the observation of the normal ABR thresholds in $S c x$-null mice without otitis media.

\section{DISCUSSION}

Our data demonstrate $S c x$ expression in the sensory epithelium of the mammalian cochlea in functionally diverse cell types. $S c x$ is expressed in interdental cells of the spiral limbus that produce otoanchorin which is defective in nonsyndromic, autosomal recessive deafness, DFNB22 (Zwaenepoel et al. 2002). Scx is found in mechanically sensitive hair cells that transduce sound energy into electrical signals that subserve hearing (Hudspeth 1997). In addition, Scx is present in Claudius cells which, along with other supporting cell types, play a role in cochlear ion homeostasis essential for mechanotransduction of sensory hair cells (Zhu and Zhao 2010). Given the functionally critical cell types in which $S c x$ is expressed, it was surprising to observe a heterogeneous auditory phenotype in $S c x$-null mice that could be explained without postulating a sensorineural etiology: otitis media was observed in $\sim 50 \%$ of mutants evaluated. Laser interferometric measurement of malleal movement in the $S c x$-null mouse with otitis media demonstrated decreased vibration of the ossicular chain that can account for the elevated auditory thresholds observed. Moreover, normal auditory thresholds in Scx-null mice without otitis media suggest that $S c x$ is not required for auditory function. However, functional redundancy cannot formally be ruled out as Paraxis (Burgess et al. 1995), another member of the small Twist family of transcription factors that is required for somite formation and musculoskeletal patterning (Burgess et al. 1996), and Hand1 and Hand2, members of the small Hand subfamily of bHLH transcription factors (Atchley and Fitch 1997; Firulli 2003) that regulate cardiac development (McFadden et al. 2005), have significant sequence similarity to $S c x$ and their expression has not been adequately defined in the mouse inner ear.

The etiology of otitis media in $S c x$-null mice is unknown. $S c x$ mutants are viable, but they display dramatic musculoskeletal defects including dorsiflexion of the forepaws, inability to reorient efficiently in response to rapidly induced postural changes, and the inability to move the tail (Murchison et al. 2007). Otitis media is more common in mice that are less physically robust and may be related to a commensurate alteration in the immune response. However, it is intriguing to consider $S c x$-mediated mechanisms for the pathogenesis of otitis media. Scx is expressed in all force bearing and anchoring tendons in the body and the musculoskeletal defects in mutant mice are attributed to failed tendon differentiation (Murchison et al. 2007). The cranial, thoracic, and abdominal musculatures are precisely coordinated during suckling and swallowing. It is conceivable that even minor alterations in tendonmediated force transmission during synchronous muscle contractions in Scx-null mice could produce extraesophageal reflux into the nasopharynx and Eustachian tube, predisposing the animal to middle ear infection. Extraesophageal reflux is a proposed mechanism by which children develop otitis media (Hall-Stoodley et al. 2006; O'Reilly et al. 2008), the most common infection in children for which antibiotics are prescribed (Coker et al. 2010). Alternatively, the musculature responsible for active dilation of the Eustachian tube (the tensor veli palatini and levator veli palatini) may not exert sufficient force to modulate the ductal valve to equalize middle ear pressures and allow fluid drainage from the middle ear mucosa to the nasopharynx. Future evaluation of the 

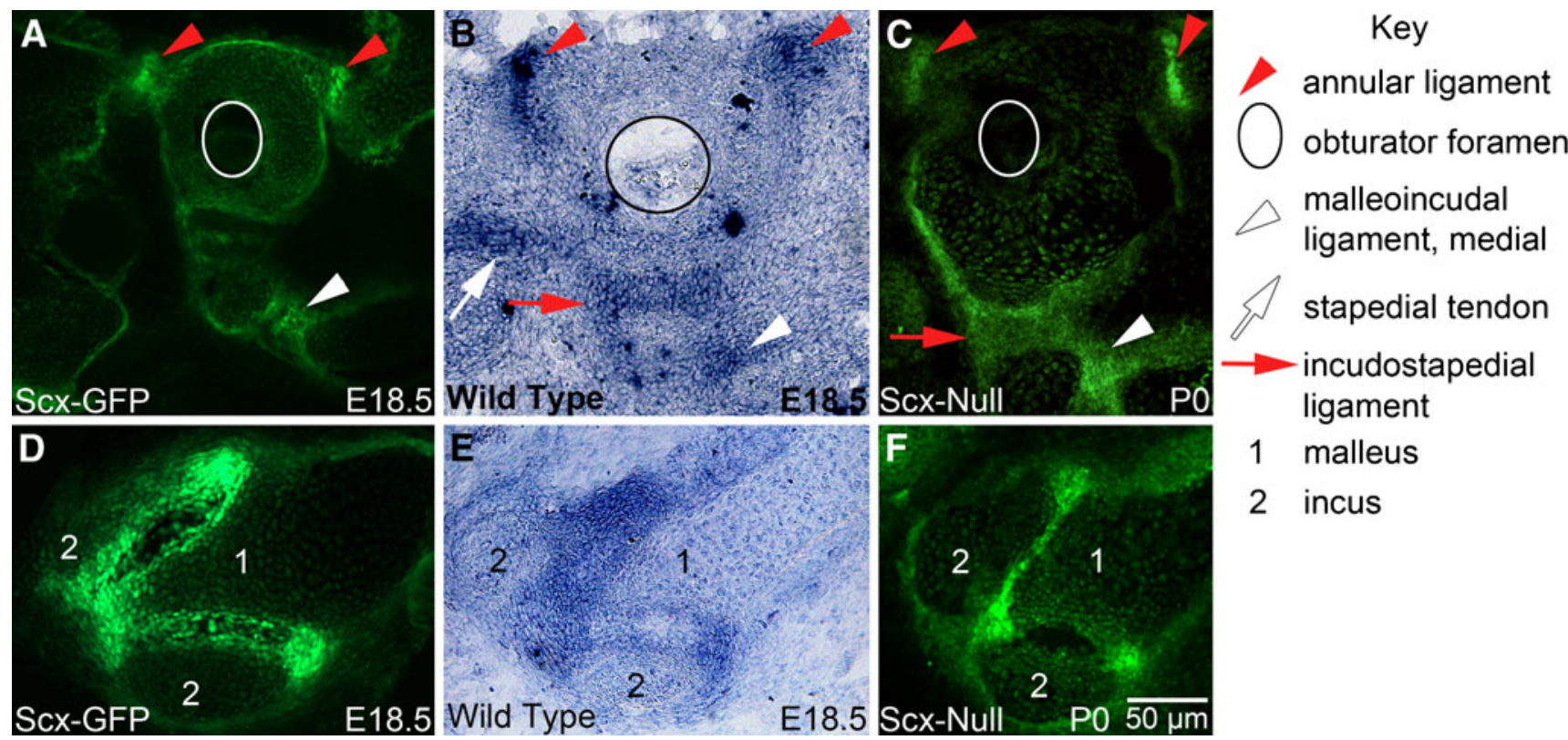

stapedial tendon

$\rightarrow$ incudostapedial ligament

1 malleus

2 incus

FIG. 7. $S_{C X}$ is expressed in the annular, incudostapedial, malleoincudal ligaments. GFP is expressed in the ligaments of the $S C X-G F P$-reporter mouse (A, D) and $S_{C X}$ mRNA is expressed in the ligaments of wild-type mice (B, E) at E18.5. GFP is expressed in ligaments of the $S_{C X}$-null middle ear $(\mathbf{C}, \mathbf{F})$ at P0 (the $S C x$-null mouse carries the $S_{C X}$-GFP reporter allele). The annular ligament (A, B, red arrowheads) secures the stapes footplate to the oval window of the cochlea. The malleoincudal ligament lines the V-shaped articulating surface between the malleus and incus (see Fig. 4A, number 6) and is presented in full cross section in (D, E; 1 malleus; 2 incus). The medial extent of the malleoincudal joint is indicated by the white arrowhead in (A, B). SCx mRNA expression in the incudostapedial ligament is indicated by the red arrow in (B). Stapedial tendon mRNA expression is indicated by the white arrow in (B) and serves as an anatomical reference. The annular (C, red arrowheads), incudostapedial (C, red arrow), and malleoincudal (C, white arrowhead and F) ligaments express SCX-GFP in the P0 SCX-null mutant. The white and black circles (A-C) outline the obturator foramen of the stapes. See the Key to facilitate comparisons. The scale bar in (F) applies to all panels.

pathogenesis of otitis media in a Scx conditional null that lacks cranial tendons in the absence of confounding musculoskeletal defects in the axial and appendicular skeleton could be informative in determining the etiology of otitis media. Established mouse models for otitis media include genetic manipulation of the immune system, inoculation of the middle ear with infectious agents or their molecular by-products, and mutants with craniofacial defects (MacArthur and Trune 2006; Ryan et al. 2006; Trune and Zheng 2009). A genetically defined mouse model of otitis media with altered muscular control of the Eustachian tube may be a valuable tool to refine the role of the musculoskeletal system in otitis media pathogenesis and for testing appropriate therapeutic interventions.

We have shown that $S c x$ labels middle ear tendon progenitors at E14.5 and differentiated tenocytes at E16.5-18.5. These observations are consistent with $S c x$ expression in tendon lineages in the axial and appendicular skeleton (Cserjesi et al. 1995; Schweitzer et al. 2001). The $S c x-G F P$ reporter will greatly facilitate analyses of tendon development in defined mouse mutants that affect middle ear formation. Furthermore, in the mature middle ear, Scx-GFP effectively illuminates the middle ear cavity with intense green fluorescence that backlights the tympanic membrane and precisely defines the ligamentous connections along the length of the ossicular chain, also high- lighting the incal and malleal associations with the walls of the middle ear cavity. We anticipate that the $S c x-G F P$ reporter will emerge as a powerful tool to advance knowledge of middle ear tendon and muscle development and enable novel imaging strategies to refine our understanding of the biomechanical function of the mouse middle ear.

The stapedius tendon in Scx-null mice displays $\mathrm{GFP}^{+}$tenocytes, is reduced in length, and secures the stapedius muscle to the stapes. The presence of a reduced stapedius tendon that is functionally competent to establish muscle insertion is similar to the phenotype of two force-bearing tendons, the extensor digiti quinti and the extensor carpi ulnaris, in the $S c x$ null forelimb (Murchison et al. 2007). These extensors display $\mathrm{GFP}^{+}$tenocytes but the tendons fail to grow to their normal size. Cell proliferation and cell death appear normal in Scx-null forelimb tendons and are not thought to play a role in establishing the reduced tendon phenotype. Rather, failed incorporation of tendon progenitors into the elongating tendon is proposed to explain the reduced, poorly organized matrix that is produced (Murchison et al. 2007). Consistent with this explanation, condensed progenitors adjacent to the posterior arch of the stapes are present at $\mathrm{P} 0$ in the $S c x$-null middle ear, though very few tenocytes are incorporated into the tendon proper as indicated by $S c x-G F P$ labeling of tenocytes and by the 
reduced tendon matrix produced. We conclude that $S c x$ activity is required for stapedius tendon differentiation and, as in one class of force-bearing tendons of the limb, acts to enable incorporation of condensed tendon progenitors into the growing tendon.

By contrast, the tensor tympani tendon in Scx-null mice presents very few $\mathrm{GFP}^{+}$tenocytes, and they are dispersed, though the tensor tympani muscle is inserted into the malleus. The paucity of $\mathrm{GFP}^{+}$ tenocytes in the $S c x-n u l l$ tensor tympani tendon is remarkably similar to the phenotype detected in the "intermuscular tendons" that connect muscle to muscle in the thorax and abdomen. For instance, $S c x-G F P^{+}$cells are not detected in the subscapularis tendon, the rectus abdominus tendon, or the middle tendon of the diaphragm in Scx-nulls (Murchison et al. 2007). However, a functional connective tissue matrix is produced in each of these muscles. Likewise, the reduced tensor tympani tendon in $S c x-n u l l$ mice is sufficient to enable muscle insertion into the malleus, though the physiological impact on the middle ear muscle reflex has not been assessed. In addition, expression of the tendon matrix proteins tensacin $\mathrm{C}$ and collagen XII is not affected in the middle tendon of the $S c x-n u l l$ diaphragm, though collagen 1 is drastically reduced (Murchison et al. 2007). Similarly, the tensor tympani tendon lacks the characteristic pearly white appearance contributed by longitudinally arrayed, parallel collagen 1 fibers that are so clearly evident in the $S c x-G F P$ tensor tympani, indicating altered tendon matrix biosynthesis. We conclude that $S c x$ activity, as in "intermuscular tendons," is required for appropriate tendon matrix biosynthesis.

The expression of $S c x$-GFP in the developing stapedius and tensor tympani tendons of Scx-null embryos labels tendon progenitors and differentiating tenocytes, indicating that, consistent with its role in the development of other tendons, $S c x$ is not required for middle ear tendon progenitor specification. We have shown, rather, that $S c x$ is required for middle ear tendon differentiation, though the underlying molecular mechanism remains elusive. In forelimb tendons of $S c x$ null embryos, collagen XIV (Gelse et al. 2003) and tenomodulin (Brandau et al. 2001) expression are lost, and collagen I levels are decreased (Murchison et al. 2007). Moreover, direct regulation of collagen I enhancers has been demonstrated (Lejard et al. 2007; Espira et al. 2009). Collagen XIV regulates the diameter of collagen fibrils (Young et al. 2002), tenomodulin subtly influences tendon proliferation (Docheva et al. 2005), and collagen $\mathrm{I}$ is the major structural protein in the collagen matrix of tendons (Orgel et al. 2006). The misregulation of these proteins in Scx-nulls is not thought to account for Scx function in tenocyte differentiation and for the range of phenotypes observed in the tendons of mutants (Murchison et al. 2007). No other genes known to be specifically expressed in tendons were altered in Scx-nulls (Murchison et al. 2007). The identification of transcriptional regulators of $S c x$ as well as those genes regulated by $S c x$ activity will be necessary to understand the transcriptional control of tendon formation.

The middle ear muscle reflex in humans acts on the stapedius and tensor tympani muscles to increase impedance (Mukerji et al. 2010). The stapedius contracts to limit movement of the stapes footplate on the oval window of the cochlea and the tensor tympani contracts and pulls the tympanic membrane inward. The resulting increased impedance of the middle ear reduces transmission of potentially damaging loud sound from the external ear canal to the inner ear. The $S c x-n u l l$ middle ear tendons are reduced in size and have altered matrix properties. We postulate that $S c x$ null mice may display an abnormal middle ear muscle reflex in response to chronic, intense suprathreshold sound stimuli and may exhibit enhanced susceptibility to noise-induced hearing loss. Middle ear muscle reflex testing in the Scx-null mouse is not the ideal model system given the systemic musculoskeletal defects and postural weakness. A conditional mouse model with $S c x$ deleted in cranial tissues would be far more efficacious to evaluate the mechanical contribution of the middle ear tendons and musculature to the acoustic reflex. In addition, a mouse predisposed to noise-induced hearing loss may serve as a useful model to discern the mechanisms by which loud sound damages sensory hair cells and to develop interventions to effectively protect auditory function.

\section{ACKNOWLEDGEMENTS}

We thank Doris K. Wu (National Institute on Deafness and other Communication Disorders [NIDCD]) for providing the Acan and Atoh1 in situ hybridization probes; Dennis R. Trune and Beth J. Kempton for expert guidance with ABRs and GMA histochemistry; Brian A. Pryce and Spencer S. Watson for assistance with $S c x$ mouse breeding; Jonathan Jungwirth for technical assistance with tissue dissection; and Patreece Suen for help with in situ hybridization. This work was supported by grants from the NIDCD and the National Institute of Arthritis and Musculoskeletal and Skin Diseases: DC R01 008595 and DC R01 008595-03S2 (to JB); P30 DC005983; R01 DC04554 (to TR); and R01 AR055640 (to RS).

\section{REFERENCES}

Atchley WR, Fitch WM (1997) A natural classification of the basic helix-loop-helix class of transcription factors. Proc Natl Acad Sci U S A 94:5172-5176

Brandau O, Meindl A, Fassler R, Aszodi A (2001) A novel gene, tendin, is strongly expressed in tendons and ligaments and 
shows high homology with chondromodulin-I. Dev Dyn 221:7280. doi:10.1002/dvdy.1126

Brown D, Wagner D, Li X, Richardson JA, Olson EN (1999) Dual role of the basic helix-loop-helix transcription factor scleraxis in mesoderm formation and chondrogenesis during mouse embryogenesis. Development 126:4317-4329

Burgess R, Cserjesi P, Ligon KL, Olson EN (1995) Paraxis: a basic helix-loop-helix protein expressed in paraxial mesoderm and developing somites. Dev Biol 168:296-306. doi:10.1006/ dbio.1995.1081

Burgess R, Rawls A, Brown D, Bradley A, Olson EN (1996) Requirement of the paraxis gene for somite formation and musculoskeletal patterning. Nature 384:570-573. doi:10.1038/ $384570 \mathrm{a} 0$

Coker TR, Chan LS, Newberry SJ, Limbos MA, Suttorp MJ, Shekelle PG, TАкATA GS (2010) Diagnosis, microbial epidemiology, and antibiotic treatment of acute otitis media in children: a systematic review. JAMA 304:2161-2169. doi:10.1001/jama.2010.1651

Cserjesi P, Brown D, Ligon KL, Lyons GE, Copeland NG, Gilbert DJ, Jenkins NA, OLson EN (1995) Scleraxis: a basic helix-loop-helix protein that prefigures skeletal formation during mouse embryogenesis. Development 121:1099-1110

Docheva D, Hunziker EB, Fassler R, Brandau O (2005) Tenomodulin is necessary for tenocyte proliferation and tendon maturation. Mol Cell Biol 25:699-705. doi:10.1128/MCB.25.2.699-705.2005

Espira L, Lamoureux L, Jones SC, Gerard RD, Dixon IM, Czubryt MP (2009) The basic helix-loop-helix transcription factor scleraxis regulates fibroblast collagen synthesis. J Mol Cell Cardiol 47:188-195. doi:10.1016/j.yjmcc.2009.03.024

Firulli AB (2003) A HANDful of questions: the molecular biology of the heart and neural crest derivatives (HAND)-subclass of basic helix-loop-helix transcription factors. Gene 312:27-40. doi: S0378111903006693

Franco HL, Casasnovas J, Rodriguez-Medina JR, Cadilla CL (2010) Redundant or separate entities?-roles of Twist1 and Twist2 as molecular switches during gene transcription. Nucleic Acids Res. doi:10.1093/nar/gkq890

Gelse K, Poschl E, Aigner T (2003) Collagens-structure, function, and biosynthesis. Adv Drug Deliv Rev 55:1531-1546. doi: S0169409X03001820

GILLESPIE PG, Muller U (2009) Mechanotransduction by hair cells: models, molecules, and mechanisms. Cell 139:33-44. doi:10.1016/j.cell.2009.09.010

Hall-Stoodley L, Hu FZ, Gieseke A, Nistico L, Nguyen D, Hayes J, Forbes M, Greenberg DP, Dice B, Burrows A, Wackym PA, Stoodley P, Post JC, Ehrlich GD, Kerschner JE (2006) Direct detection of bacterial biofilms on the middle-ear mucosa of children with chronic otitis media. JAMA 296:202-211. doi:10.1001/jama.296.2.202

He W, Fridberger A, Porsov E, Grosh K, Ren T (2008) Reverse wave propagation in the cochlea. Proc Natl Acad Sci U S A 105:27292733. doi: $10.1073 /$ pnas. 0708103105

Helms AW, Johnson JE (1998) Progenitors of dorsal commissural interneurons are defined by MATH1 expression. Development 125:919-928

HudsPETH AJ (1997) How hearing happens. Neuron 19:947-950. doi:S0896-6273(00)80385-2

Hwang CH, Wu DK (2008) Noggin heterozygous mice: an animal model for congenital conductive hearing loss in humans. Hum Mol Genet 17:844-853. doi:10.1093/hmg/ddm356

Kelley MW, Driver EC, Puligilla C (2009) Regulation of cell fate and patterning in the developing mammalian cochlea. Curr Opin Otolaryngol Head Neck Surg 17:381-387. doi:10.1097/ MOO.0b013e3283303347

Lejard V, Brideau G, Blais F, Salingcarnboriboon R, Wagner G, Roehrl MH, Noda M, Duprez D, Houillier P, Rossert J (2007) Scleraxis and NFATc regulate the expression of the pro-alphal
(I) collagen gene in tendon fibroblasts. J Biol Chem 282:1766517675. doi:10.1074/jbc.M610113200

MacArthur CJ, Trune DR (2006) Mouse models of otitis media. Curr Opin Otolaryngol Head Neck Surg 14:341-346. doi:10.1097/01. moo.0000244193.97301.d700020840-200610000-00011

MaLlo M (2001) Formation of the middle ear: recent progress on the developmental and molecular mechanisms. Dev Biol 231:410-419. doi:10.1006/dbio.2001.0154S0012-1606(01)90154-X

MALLo M (2003) Formation of the outer and middle ear, molecular mechanisms. Curr Top Dev Biol 57:85-113

McFadden DG, Barbosa AC, Richardson JA, Schneider MD, SRivastava D, Olson EN (2005) The Hand1 and Hand2 transcription factors regulate expansion of the embryonic cardiac ventricles in a gene dosage-dependent manner. Development 132:189-201. doi:10.1242/dev.01562

Mitchell C, Kempton JB, Creedon T, Trune D (1996) Rapid acquisition of auditory brainstem responses with multiple frequency and intensity tone-bursts. Hear Res 99:38-46

MukerJi S, Windsor AM, LeE DJ (2010) Auditory brainstem circuits that mediate the middle ear muscle reflex. Trends Amplif 14:170-191. doi:10.1177/1084713810381771

Murchison ND, Price BA, Conner DA, Keene DR, Olson EN, Tabin CJ, Schweitzer R (2007) Regulation of tendon differentiation by scleraxis distinguishes force-transmitting tendons from muscleanchoring tendons. Development 134:2697-2708. doi:10.1242/ dev.001933

O'Reiliy RC, He Z, Bloedon E, Papsin B, Lundy L, Bolling L, Soundar S, Cook S, Reiliy JS, Schmidt R, Deutsch ES, Barth P, Mehta DI (2008) The role of extraesophageal reflux in otitis media in infants and children. Laryngoscope 118:1-9. doi:10.1097/ MLG.0b013e31817924a300005537-200807001-00001

Orgel JP, Irving TC, Miller A, Wess TJ (2006) Microfibrillar structure of type I collagen in situ. Proc Natl Acad Sci U S A 103:9001-9005. doi:10.1073/pnas.0502718103

Pryce BA, Brent AE, Murchison ND, Tabin CJ, Schweitzer R (2007) Generation of transgenic tendon reporters, ScxGFP and ScxAP, using regulatory elements of the scleraxis gene. Dev Dyn 236:1677-1682. doi:10.1002/dvdy.21179

REN T (2004) Reverse propagation of sound in the gerbil cochlea. Nat Neurosci 7:333-334. doi:10.1038/nn1216nn1216

Ryan AF, Ebmeyer J, Furukawa M, Pak K, Melhus A, Wasserman SI, Chung WH (2006) Mouse models of induced otitis media. Brain Res 1091:3-8. doi:10.1016/j.brainres.2006.02.004

SANDELl LJ, MoRRIS N, RoBbins JR, GoLDRING MB (1991) Alternatively spliced type II procollagen mRNAs define distinct populations of cells during vertebral development: differential expression of the amino-propeptide. J Cell Biol 114:1307-1319

Schweitzer R, Chyung JH, Murtaugh LC, Brent AE, Rosen V, Olson EN, Lassar A, Tabin CJ (2001) Analysis of the tendon cell fate using Scleraxis, a specific marker for tendons and ligaments. Development 128:3855-3866

TRUNE DR, ZHENG QY (2009) Mouse models for human otitis media. Brain Res 1277:90-103. doi:10.1016/j.brainres.2009.02.047

Young BB, Zhang G, Koch M, Birk DE (2002) The roles of types XII and XIV collagen in fibrillogenesis and matrix assembly in the developing cornea. J Cell Biochem 87:208-220. doi:10.1002/jcb.10290

Zhu Y, Zhaо HB (2010) ATP-mediated potassium recycling in the cochlear supporting cells. Purinergic Signal 6:221-229. doi:10.1007/s11302-010-9184-9

Zwaenepoel I, Mustapha M, Leibovici M, Verpy E, Goodyear R, Liu XZ, Nouaille S, Nance WE, Kanaan M, Avraham KB, Tekaia F, Loiselet J, Lathrop M, Richardson G, Petit C (2002) Otoancorin, an inner ear protein restricted to the interface between the apical surface of sensory epithelia and their overlying acellular gels, is defective in autosomal recessive deafness DFNB22. Proc Natl Acad Sci U S A 99:6240-6245. doi:10.1073/ pnas.082515999082515999 